\title{
Blood Immunoglobulins, Complement and TNF receptor following Minimally Invasive Surgery in Patients Undergoing Pulmonary Lobectomy
}

H Anne Leaver ${ }^{\mathrm{a}}$, Stewart R Craig ${ }^{\mathrm{b}}$, Dino Rotondo ${ }^{\mathrm{c}}$, and William S Walker ${ }^{\mathrm{d}}$

a Cell Biology R\&D, SNBTS \& Clinical Neurosciences Edinburgh University, b Department of Cardiothoracic Surgery, Golden Jubilee Hospital, Glasgow, ${ }^{\mathrm{c}}$ Strathclyde Institute of Pharmacy \& Biomedical Sciences, Strathclyde University, Glasgow, and d Division of Cardiothoracic Surgery, Royal Infirmary, Edinburgh

Correspondence to: $\quad$ Dr HA Leaver, Cell Biology Laboratory, SNBTS, 21 Ellen's Glen Road, Edinburgh EH177GT, UK.

Telephone Number: ～(UK)-131-314-5680 Fax: (UK)-131-536-6764

e-mail: hanne.leaver@gmail.com

Running Title:

Blood Immunoglobulins, Complement and TNF receptor following Minimally Invasive Surgery (10 words)

Keywords: Mediators, Minimally Invasive Pulmonary Lobectomy (5 words) 
The reasons for improved survival following minimally invasive surgery remain elusive. Circulating mediators link surgical trauma, vascular and tissue homeostasis. Acute phase reactants, leukocytes and leukocyte Reactive Oxygen Species (ROS) are affected differentially by minimally invasive videoassisted thoracic surgery (VATS). Also, immunoglobulins, complement, TNF receptor and P-selectin changes have been observed, but the influence of minimally invasive surgery on these opsonins is less well defined. In this prospective randomised trial, 41 patients were randomly assigned to minimally invasive or open thoracic surgery, and immunoglobulins and vascular endothelial damage biomarkers were analysed. Humoral mediators (blood IgG, IgM, IgA; complement fragments C3, C4, and complement haemolytic index of activation CH50; TNF receptors I, II and P-selectin) were analysed before and 2, 5 and 7 days after surgery. Post-surgical changes in individual patients were determined.

Substantial immunoglobulin decreases followed minimally invasive and open surgery. Decreased IgG, $\operatorname{IgM}$ and $\operatorname{IgE}$ were detected 2 days after surgery, and IgG and IgM after 7 days. These changes were greater than haemodilution, reaching greater significance in open surgery patients. Immunoglobulin decreases followed lymphocyte decreases. In contrast, increased complement and inflammatory endothelial cell signals (C3 and C4, soluble TNFR-II) were detected 7 days after surgery. In both groups, increased C3 and TNFR-II followed early acute phase reactants CRP, IL-6 and ROS. Acute phase reactants and CD4/CD8 lymphocytes were factors most attenuated in patients undergoing minimally invasive thoracic surgery (VATS). This study suggests local trauma mediators are better biomarkers than circulating opsonins in defining the response to minimally invasive surgery, and a systems approach, comparing individual metabolic responses, is effective in small patient groups.

\section{INTRODUCTION}

Major cardiothoracic surgery is associated with release of local and systemic mediators, acting on vascular and pulmonary systems which may be compromised by underlying disease [1]. Although improvements in survival and infection rates have been reported following minimally invasive surgery, the underlying mechanisms remain poorly defined. Following surgery, there have been reports of improved outcomes of minimally invasive techniques on immunosuppression, measured as infection rates, circulating lymphocytes and immunoglobulins [2-6]. Circulating immunoglobulins play an important part in immunity and immunoglobulin preparations have been used for prophylaxis for patients at high risk from sepsis [7, 8]. However, there has been little analysis of whether differences in post surgical immunoglobulins are due to changes in immunoglobulin turnover or blood loss. Also, prospective randomised trials of minimally invasive surgery have become more difficult to perform, as the use of conventional open surgery has decreased and minimally invasive approaches have become the method of choice. In the current randomised prospective study of minimally invasive pulmonary 
lobectomy, humoral opsonins, including immunoglobulins, complement, selectins and TNF binding proteins were analysed, to determine immunological and humoral biomarkers following surgery.

It is well known that the cytokine network is closely linked to inflammatory and vascular signals, and emerging evidence suggest that it undergoes specific changes following minimally invasive surgery. IL-6 is a characteristic marker of minimally invasive surgery. IL-6 levels increased by up to 18 -fold within two days of major surgery [9]. We previously detected lower IL-6 release in VATS, compared with conventional open surgery in the patient group used in this study following pulmonary surgery [10]. Characteristic IL-6 changes were also detected in other sites of minimally invasive surgery. In a study of laparoscopic excisions for rectal cancer, IL-6 decreased 1 and 5 days after laparoscopic surgery, but increased after open surgery, but not TNF [11]. In the patient group covered by this study we also reported that TNF was unchanged, and in this paper we describe post surgical changes in circulating TNF binding proteins which may neutralise locally released TNF. We also report post surgical changes in opsonins that link humoral mediators including IL-6 and lung tissue immunity: selectins and complement. The selectins link stress signals, including NF-KB and ATF-2 factors, to vascular responses. P-, E-, and L-selectin recognize leukocyte and endothelial glycoproteins and Pselectin is elevated in patients with haemolytic disease and lung cancer [12-14]. Complement and selectins also act as acute phase mediators [14]. Complement serum proteins, especially C3 and C4, may be produced by a wide variety of tissues and circulating immune cells such as macrophages, although the greatest amount originates from the liver [15]. C3 and C4, especially, are associated with elevated IL-6, which stimulates renal acute phase protein production [16]. Elevated haemolytic complement activity (CH50), a biomarker of haemolytic complement in the classical pathway, is associated with cancer, infection and conditions associated with chronic inflammation [15]. Therefore, in this study, the role of these circulating opsonins in linking immunological and vascular responses (CH50 and P-selectin) were investigated.

The immunoglobulins are major humoral mediators, playing a central role in immune surveillance and also a local role in lung pathophysiology. This study investigates the impact of pulmonary surgery on circulating immunoglobulins. The lung is a site of specific anaphylactic responses and of immunosurveillance. In the patient group used in this study, we previously reported differential changes in immunoglobulin producing B cells and $\mathrm{T}$ cells controlling the immune response following minimally invasive surgery [3]. Additionally, oxygen tension in pulmonary tissue is high, and changes in oxidative metabolism were detected in circulating leukocytes, which differed in VATS patients [3, 10]. However, while there have been recent advances in understanding ROS bioactivity at a cellular level, less is known about their activity in integrated whole body network responses. In the current study, changes in immunoglobulins, complement, selectins and TNF binding proteins were analysed in individual patients. These changes were compared with previously identified cellular and humoral biomarkers (ROS and acute phase mediators), to investigate potential network and mediator interactions during the post-operative period. 


\section{MATERIALS AND METHODS}

In this communication, we present previously unpublished data obtained from a prospective randomised trial, analysing peripheral venous blood samples collected during a study of acute phase [10] and leukocyte [3] changes following minimally invasive or conventional surgery for brochogenic carcinoma. The numbers of patients in each group was calculated from previous reports of leukocyte changes after surgery [3] derived from post surgical leucocyte ranges reported in laparoscopic surgery trials, and patient inclusion criteria have been described $[3,10]$. To summarize, patients with peripheral pulmonary opacity consistent with peripheral brochogenic carcinoma underwent standard surgical assessment and staging by bronchoscopy, mediastinoscopy and computed axial tomography of thorax and upper abdomen. Routine haematological and biochemical analysis (Haemoglobin, urea) and blood loss analysis was performed to monitor haemodilution following surgery. Local ethical committee permission was obtained, and patients scheduled to undergo pulmonary lobectomy were randomly allocated to VATS or open surgery. There was no significant difference in age or histological diagnosis between the two study groups: Median age was 62 (47-74) in open surgery, compared with 64.5 (4678) in VATS patients; sex ratio 14/5 (open surgery) and 8/14 M/F (VATS). Clinical outcomes have been described in detail [10], 'duration of surgery, length of stay and pathology in the two groups were not significantly different. Complications were: one patient with intrapleural adhesions, bleeding and lung collapse, and one patient with poor respiratory function in the VATS group; three cases of pulmonary infection, and one case of cardiac dysrythmia in the open group.

The VATS technique involved $4-5 \mathrm{~cm}$ submammary incision over the postero-lateral chest wall. The open approach involved limited thoracotomy, avoiding trapezius and rhomboid muscles, but divided the latissimus dorsi and the posterior third of serratus anterior. Both techniques involved initial identification of the pulmonary artery and division of fused fissures, then dissection and division of hilar structures. Equivalent nodal dissection was carried out in open and VATS patients and anaesthetic and analgesic regimens did not differ between groups. Post-operative pain control was managed with patient-controlled morphine administration, supplemented by intercostal nerve blocks as required and oral analgesia. No non-steroidal anti-inflammatory drugs or blood transfusion (which is associated with immunosuppression) was used. Fasting venous blood samples were obtained pre-operatively and at 2 and 7 days post-operatively from 41 patients allocated randomly to alternative study groups at the time of surgery. Inflammatory/ endothelial biomarkers were analysed in a smaller cohort of 23 patients as an additional preliminary study, with more frequent sampling in order to determine the time course of any post surgical changes. There was no selection or exclusion of data for any patients or markers and the individuals involved in analysis were unaware of the operative technique used.'

Laser nephelometry was used to measure $\operatorname{IgG}, \operatorname{IgM}, \operatorname{IgA}, \mathrm{C} 3, \mathrm{C} 4[17,18]$. Haemolytic activity of complement (CH50), used haemolysis of sheep erythrocytes sensitized by $\mathrm{C} 3, \mathrm{C} 4$ specific antibodies (One CH50 unit: volume of serum lysing 50\% erythrocytes). P-selectin TNFR-I and -II were analysed 
using monoclonal antibodies supplied by British Biotechnology, London UK and Monosan, Brussels, Belgium, respectively based ELISA, as described previously [10]. The change in opsonins and mediators after surgery was calculated as percent of pre-surgical levels within each patient. Results are expressed as median or mean \pm SEM. Results significantly different from pre-surgical levels were analysed using the Wilcoxon matched pairs signed two tailed rank test, comparing pre-operative and post-operative opsonin levels within the same patient. Significantly different results were dentoted * $\mathrm{p}<0.05, * * \mathrm{p}<0.005$ and $* * * \mathrm{p}<0.0005$. The correlation between changes in related parameters after surgery was analysed using simple linear regression analysis. Patients with malignant histology predominated in both open and VATS study groups. There were 18 patients with malignancy in the open surgery group and 18 patients with malignant histology in the VATS group.

Table 1. Immunoglobulin depletion and Complement activation following Conventional or Minimally Invasive Surgery. Median concentrations in plasma \pm SEM in IgG, IgM and IgA in 19 patients undergoing Open Surgery and 22 Minimally Invasive Surgery (VATS) patients, before (day 0) and days 2 and 7 post-op. * results significantly $\mathrm{p}<0.05$ different from paired pre-surgical (day 0 ) levels in same patient; *, p <0.05; **, $\mathrm{p}<0.005 ; * * *, \mathrm{p}<0.0005$. $^{*}$, and indicators of haemodilution (Haemoglobin $\mathrm{Hb}$ ) and renal function (Urea) day 0 , day 1 post-op, median + SEM.

\begin{tabular}{|c|c|c|c|c|}
\hline \multicolumn{5}{|c|}{ Open Surgery } \\
\hline Days & Post-Op & 0 & 2 & 7 \\
\hline $\operatorname{IgG}$ & $\mathrm{g} \mathrm{L}^{-1} \times 100$ & $10.6 \pm 0.57$ & $8.36 \pm 0.52 * * *$ & $9.57 \pm 0.57 * *$ \\
\hline $\operatorname{IgM}$ & $\mathrm{g} \mathrm{L}^{-1} \times 10$ & $1.1 \pm 0.14$ & $0.79 \pm 0.15 * * *$ & $0.93 \pm 0.14 * *$ \\
\hline $\operatorname{IgA}$ & $\mathrm{g} \mathrm{L}^{-1} \times 10$ & $2.42 \pm 0.25$ & $2.04 \pm 0.28 * * *$ & $2.4 \overline{4}+0.34$ \\
\hline C3 & $\mathrm{g} \mathrm{L}^{-1}$ & $1.19 \pm 0.07$ & $1.26 \pm 0.06$ & $1.53 \pm 0.06^{* *}$ \\
\hline $\mathrm{C} 4$ & $\mathrm{~g} \mathrm{~L}^{-1}$ & $0.3 \pm 0.02$ & $0.34 \pm 0.02$ & $0.39 \pm 0.02 * *$ \\
\hline CH50 & $\mathrm{U}$ & $84 \pm 34.4$ & $76 \pm 6.3 *$ & $109 \pm 8.7$ \\
\hline $\begin{array}{l}\mathrm{Hb}^{\ddagger} \\
\text { Urea }^{¥}\end{array}$ & $\begin{array}{c}\mathrm{g} / \mathrm{dL} \\
\mathrm{mMol} \mathrm{L}^{-1}\end{array}$ & $\begin{array}{c}14.0 \pm 0.5 \\
4.45 \pm 0.57\end{array}$ & $\begin{array}{c}13.0 \pm 0.6 \\
5.05 \pm 0.52\end{array}$ & \\
\hline \multicolumn{5}{|c|}{ Minimally Invasive Surgery } \\
\hline Days & Post-Op & 0 & 2 & 7 \\
\hline $\operatorname{IgG}$ & $\mathrm{g} \mathrm{L}^{-1} \times 100$ & $9.89 \pm 0.61$ & $8.76 \pm 0.57 * * *$ & $9.13 \pm 0.65 * *$ \\
\hline IgM & $\mathrm{g} \mathrm{L}^{-1} \times 10$ & $0.77 \pm 0.11$ & $0.63 \pm 0.10^{*}$ & $0.8 \overline{6} \pm 0.12$ \\
\hline $\operatorname{IgA}$ & $\mathrm{g} \mathrm{L}^{-1} \times 10$ & $2.36 \pm 0.35$ & $2.29 \pm 0.33 * * *$ & $2.33 \pm 0.38$ \\
\hline $\mathrm{C} 3$ & $\mathrm{~g} \mathrm{~L}^{-1}$ & $1.24 \pm 0.06$ & $1.22 \pm 0.04$ & $1.62 \pm 0.07 * * *$ \\
\hline $\mathrm{C} 4$ & $\mathrm{~g} \mathrm{~L}^{-1}$ & $0.33 \pm 0.02$ & $0.31 \pm 0.16$ & $0.43 \pm 0.02 * * *$ \\
\hline $\mathrm{CH} 50$ & $\mathrm{U}$ & $73 \pm 11.1$ & $68 \pm 10.1$ & $70 \pm 10.2$ \\
\hline $\mathrm{Hb}^{¥}$ & $(\mathrm{~g} / \mathrm{dL})$ & $13.5 \pm 0.5$ & $12.5 \pm 0.6$ & \\
\hline Urea $^{¥}$ & $\mathrm{mMol} \mathrm{L}^{-1}$ & $4.1 \pm 0.33$ & $4.3 \pm 0.28$ & \\
\hline
\end{tabular}




\section{RESULTS}

Changes in Immunoglobulins following Surgery

Substantial decreases in venous IgG, IgM and IgA were detected after surgery in patients undergoing pulmonary lobectomy (Table 1). In pre-operative patients, a relatively wide range of immunoglobulin levels were detected, which overlapped with postoperative immunoglobulin levels (Fig 1) and therefore post-operative changes in individual patients were analysed, as these changes were the focus of this study. IgG, IgM, IgA decreases ( $₫$ SEM) were: IgG $21 \pm 3 \%(18 \pm 2 \%), \operatorname{IgM} 13 \pm 3 \%(6 \pm 10 \%), \operatorname{IgA}$ decreased by $18 \pm 4 \%(12 \pm 2 \%)$ in open and (VATS) patients 48 hours after surgery, and decreased $13 \pm 3 \%(12 \pm 3 \%) \operatorname{IgG}$, was detected one week post-operatively (Fig $2 \mathrm{~A})$. IgM also significantly decreased one week postoperatively in open surgery patients, but not in VATS patients (Fig 2B). IgG, IgM and IgA were significantly different from pre-operative immunoglobulins, 2 days after surgery. Postoperative changes in IgM reached greater significance in open surgery patients.

Fig 1. Immunoglobulin G after Conventional Open Surgery or Minimally Invasive Surgery. Venous IgG in patients undergoing (A) conventional Open surgery (B) Minimally invasive surgery (VATS). Samples were taken pre-operatively $(0)$ and 2 and 7 days after surgery. 
Fig 2. Post-operative Changes in plasma $\operatorname{IgG}, \operatorname{IgM}$ and $\operatorname{IgA}$ after Conventional Open or Minimally Invasive Surgery. Open Surgery (dashed) Minimally invasive, VATS (solid lines). Results are median \pm SEM percentage change within the same patient in (A) $\operatorname{IgG}$, (B) $\operatorname{IgM}$ and (C) $\operatorname{IgA}$ in 19 patients undergoing open surgery and 22 VATS patients. Asterisks denote results significantly different from pre-operative immunoglobulins, ${ }^{*} \mathrm{p}<0.05 ; * * \mathrm{p}<0.005 ; * * *, \mathrm{p}<0.0005$.

Haemodilution was $9.9 \pm 3.1 \%$ in open surgery and $9.0 \pm 2.1 \%$ in minimally invasive Surgery (VATS), and median blood loss was $250 \mathrm{ml}$ (range 100ml-2.1L) in open surgery patients and 63ml (range 0$220 \mathrm{ml}$ ) in VATS patients. Thus haemodilution was lower than the $12 \%-21 \%$ decrease in immunoglobulin concentration following surgery. Therefore, this study suggested that haemodilution only contributed partially (25-50\%), to immunoglobulin depletion. Other effects of surgery may include effects on immunoglobulin production and consumption, and evidence for the former was obtained from lymphocyte analysis following surgery.

Evidence for an effect of minimally invasive surgery on immunoglobulin production in the patient group used in this study included a substantial decrease in lymphocytes following surgery [3]. The kinetics of immunoglobulin (Fig 2) and lymphocyte decreases were similar: both circulating immunoglobulins and lymphocyte numbers decreased two days after surgery. Immunoglobulin decreases were more significant in patients undergoing open surgery (Table 1 and Fig 2). Decreases in immunoglobulin producing cells and T cells were previously reported in this patient group (B cell numbers decreased by $22 \%$ in open surgery, and $18 \%$ in VATS patients two days after surgery), and even greater decreases were observed in CD4 T helper cells (32\% after open surgery, 22\% in VATS) [3]. 
Pulmonary surgery was associated with increased complement release, detected as circulating C3 and C4 one week after surgery (Table 1, Fig 3A). C3 and C4 increased 29+9\% 7 days after surgery $(\mathrm{P}<0.005$, paired t-test, comparing pre- and post-surgical $\mathrm{C} 3, \mathrm{C} 4$ within same patient). There was evidence of reduced C4 release in VATS $(31 \pm 11 \%$ open surgery; compared with $22 \pm 6 \%$ in VATS patients). The complement increase occurred later than immunoglobulin changes, which were significantly greater on day 2 after surgery (Fig 2), and there was evidence of reduced C4 release in VATS patients (Fig 3A). Increased post-operative C3 and C4 in VATS and open surgery could indicate a slow onset "acute-phase" response. This response may be partially inflammatory, as complement haemolytic index of activation (CH50) indicated end-activation of complement at day 7 in open surgery, but not in VATS patients (Table 1). Proteolytic cleavage of C3 or C4 during complement activation depletes serum $\mathrm{C} 3$ and $\mathrm{C} 4$. The high correlation between $\mathrm{C} 3$ and $\mathrm{C} 4$ changes indicated a possible common clearance pathway in thoracotomy patients.

Table 2. Inflammatory/Endothelial Biomarker increases after Conventional or Minimally Invasive Surgery. Results are median concentration, $\mathrm{ng} / \mathrm{ml}$, in plasma samples, analysed in 12 patients undergoing open surgery and 11 VATS patients, taken before (0 days post-op and on days 2 and 7 post-op. The number of patient samples analysed at each time point are shown in parenthesis * results significantly $\mathrm{p}<0.05$ different from paired pre-surgical (day 0 ) levels in same patient; * $\mathrm{p}<0.05 ; * *$, $\mathrm{p}<0.005 ; * * *, \mathrm{p}<0.0005$.

\begin{tabular}{|c|c|c|c|c|c|c|c|}
\hline \multicolumn{8}{|c|}{ Open Surgery } \\
\hline $\begin{array}{l}\text { Hours } \\
\text { Post-Op }\end{array}$ & 0 & 4 & 24 & 48 & 72 & 120 & 168 \\
\hline TNFR-II & $\begin{array}{c}3.82+0.1 \\
(12)\end{array}$ & $\begin{array}{c}3.87+0.1 \\
(11)\end{array}$ & $\begin{array}{c}3.75+0.16 \\
(12)^{*}\end{array}$ & $\begin{array}{c}4.3+0.14 \\
(11) * *\end{array}$ & $\begin{array}{c}3.65+0.21 \\
(8)\end{array}$ & $\begin{array}{c}5.21+0.25 \\
(6)\end{array}$ & $\begin{array}{c}4.72+0.14 \\
(11) *\end{array}$ \\
\hline TNFR-I & $\begin{array}{c}0.82 \pm 0.1 \\
(12)\end{array}$ & $\begin{array}{c}0.69 \pm 0.11 \\
(11)\end{array}$ & $\begin{array}{c}0.89 \pm 0.13 \\
(12)^{*}\end{array}$ & $\begin{array}{c}0.86 \pm 0.13 \\
(11) * * *\end{array}$ & $\begin{array}{c}1.07 \pm 0.18 \\
(8)^{*}\end{array}$ & $\begin{array}{c}1.21 \pm 0.17 \\
(6)^{*}\end{array}$ & $\begin{array}{c}1.01 \pm 0.18 \\
(11) * * *\end{array}$ \\
\hline P-selectin & $\begin{array}{c}121 \pm 25.1 \\
(12)\end{array}$ & $\begin{array}{c}118+39.7 \\
(11)\end{array}$ & $\begin{array}{c}139 \pm 27.7 \\
(12)\end{array}$ & $\begin{array}{c}144 \pm 19.5 \\
(11)\end{array}$ & $\begin{array}{c}151 \pm 25 \\
(8)\end{array}$ & $\begin{array}{c}158 \pm 63.4 \\
(6)\end{array}$ & $\begin{array}{c}150 \pm 34.4 \\
(11)\end{array}$ \\
\hline \multicolumn{8}{|c|}{ Minimally Invasive Surgery } \\
\hline $\begin{array}{l}\text { Hours } \\
\text { Post-Op }\end{array}$ & 0 & 4 & 24 & 48 & 72 & 120 & 168 \\
\hline TNFR-II & $\begin{array}{c}3.61 \pm 0.13 \\
(11)\end{array}$ & $\begin{array}{c}4.42 \pm 0.14 \\
(10)\end{array}$ & $\begin{array}{c}4.19 \pm 0.13 \\
(11)\end{array}$ & $\begin{array}{c}4.31 \pm 0.19 \\
(11)\end{array}$ & $\begin{array}{c}3.77 \pm 0.47 \\
(8)\end{array}$ & $\begin{array}{c}3.81+0.35 \\
(6)\end{array}$ & $\begin{array}{c}5.81 \pm 0.2 \\
(10) *\end{array}$ \\
\hline TNFR-I & $\begin{array}{c}0.94+0.09 \\
(12)\end{array}$ & $\begin{array}{c}1.07 \pm 0.11 \\
(12)\end{array}$ & $\begin{array}{c}1.19 \pm 0.14 \\
(12) * *\end{array}$ & $\begin{array}{c}1.53 \pm 0.17 \\
(11) * * *\end{array}$ & $\begin{array}{c}1.39 \pm 0.19 \\
(9) *\end{array}$ & $\begin{array}{c}1.17 \pm 0.17 \\
(6)\end{array}$ & $\begin{array}{c}1.74 \pm 0.14 \\
(12) * *\end{array}$ \\
\hline P-selectin & $\begin{array}{c}83.6+42.7 \\
(12)\end{array}$ & $\begin{array}{c}74.0+37.7 \\
(12)\end{array}$ & $\begin{array}{c}40.4 \pm 20.6 \\
(12) *\end{array}$ & $\begin{array}{c}48.3 \pm 24.7 \\
(11)\end{array}$ & $\begin{array}{c}83.8+42.8 \\
(9)\end{array}$ & $\begin{array}{c}62.1+31.7 \\
(6)\end{array}$ & $\begin{array}{c}67.5 \pm 34.5 \\
(12)\end{array}$ \\
\hline
\end{tabular}

In this preliminary study of inflammatory and endothelial biomarker changes, we detected gradual increases 2-7 days after thoracotomy (Table 2). In this small study group, no significant early changes in these markers were detected in the immediate post surgical period, in samples taken $4 \mathrm{~h}$ after surgery, but changes were detected at 2-7 days after surgery. Soluble human TNFR type I/II increased after 
surgery and similar TNFR-I increases were detected in both surgical groups. However, increased TNFR-II (linked to immunomodulation) was detected in open thoracotomy (but not VATS) patients $48 \mathrm{~h}$ and $120 \mathrm{~h}$ post-op (Fig 3B). Pre-operative TNFR-II levels in our study fell within the normal range, $121 \pm 84 \mathrm{ng} / \mathrm{ml}$. It was also observed that P-selectin decreased $24-48 \mathrm{~h}$ post-op in VATS patients, whereas a gradual increase (of up to $60 \%$ ), was detected in open surgery patients [12, 14].

Fig 3. Post-operative Changes in plasma C4, and TNFR-II after Conventional and Minimally Invasive Surgery. Open Surgery (dashed) and minimally invasive surgery VATS (solid lines). Results show median \pm SEM percentage change in same patient in (A) C4 in 19 patients undergoing open surgery and 22 VATS patients. (B) TNFR-II in 12 patients undergoing open surgery and 11 VATS patients. Results significantly different from pre-operative values (day 0 ), ${ }^{*} \mathrm{p}<0.05 ;{ }^{* *} \mathrm{p}<0.005 ;{ }^{* * *}, \mathrm{p}<0.0005$.

\section{DISCUSSION}

The period immediately following surgery is important for survival. Acute phase and local mediators of surgical trauma influence immunity, vascular and pulmonary function $[10,11]$. These mediators are involved in the response to infection and longer term processes, including metastatic growth [19]. While minimally invasive surgery is associated with improved morbidity and mortality [2], the underlying mechanisms are complex and involve humoral and cellular networks of the immune and vascular sytem [20]. The aim of this study was to analyse post-surgical immunoglobulins, complement and other opsonins, to find whether minimally invasive surgery affected these mediators, and to relate these changes to other biomarkers of immune function and surgical trauma. Changes in opsonins and 
inflammatory/endothelial biomarkers were analysed within individual patients in a randomised prospective study of 41 patients undergoing pulmonary lobectomy. Substantial changes in opsonins were detected after surgery, but differences between surgical groups were small, suggesting that minimally invasive surgery had less impact on opsonisation than on acute phase changes in these patients.

Immunoglobulins play an important part in immunity. Venous IgG, IgM and IgA decreased substantially following surgery (Table 2). Both circulating immunoglobulin (Fig 2) and lymphocyte numbers [3] decreased two days after surgery, and immunoglobulin decreases were more significant in patients undergoing open surgery (Fig 2). These immunoglobulin decreases were greater than the haemodilution observed in these patients (Table 1), and immunoglobulin differences between surgical groups were also greater than haemodilution differences. We previously reported decreases in immunoglobulin producing B cells and T cells in the patient group used in this study and also differences between lymphocyte counts in open and minimally invasive patients [3]. A similar pattern of immunoglobulin and lymphocyte changes were detected following laporoscopic cholecytstectomy, with lymphocyte changes that were greater than blood immunoglobulin changes [21]. Also, we observed that lymphocyte ROS, which influence lymphocyte proliferation and apoptosis [22], were lower in VATS patients [3, 4, 20] and see this edition of Current Metabolomics, Leaver et al (2015) Cellular and Humoral Responses following Minimally Invasive Surgery: Role of Reactive Oxygen Species, in which we propose that ROS may mediate many effects of surgical stress.

As other indicators of surgical stress had previously been analysed in this patient group [3, 10], it was possible to compare these biomarkers with the opsonins analysed in the present study. Correlations between immunoglobulin and other biomarkers of open and minimally invasive surgery are shown in Fig 4. On the left side of each dendrogram, IgG changes are compared with indicators of haemodilution and $\mathrm{T}$ lymphocytes, on the right are highlighted changes in local signalling mediators (ROS), including lymphocyte ROS.

Fig 4. Dendrograph indicating links between Humoral and Cellular Mediators following Conventional Open or Minimally Invasive Surgery. The correlation between changes in related parameters after surgery was analysed using linear regression, and the length of individual branches denotes the correlation coefficient $\mathrm{r}$ for paired haematological, immunological and metabolic markers following surgery. This figure shows the correlation between post-surgical changes (dashed lines: negative, solid lines: positive) in 41 patients undergoing pulmonary lobectomy, comparing humoral, haematological and immunological factors in A) Open surgery, and B) VATS patients. Urea and blood loss (BL) $24 \mathrm{~h}$ after surgery negatively correlated with Haemoglobin $(\mathrm{Hb})$. Also, close correlations were observed between IgG and CD4 B lymphocytes in both surgical groups 2 days post surgery. In contrast, the correlation between Haemoglobin and IgG changes 2 days after open surgery was attenuated in VATS patients. Correlations between IgG and IgA changes indicated similar effects of VATS and open surgery, 2 days after surgery. On the right, negative correlations between lymphocyte 
number/lymphocyte ROS were detected 2 days after open surgery, but a positive correlation was detected in VATS patients. Differences between VATS/open surgery were detected in monocyte (mROS) and neutrophil (nROS), compared with Complement (C3 and C4) 7 days after surgery.

\section{A Open Surgery}

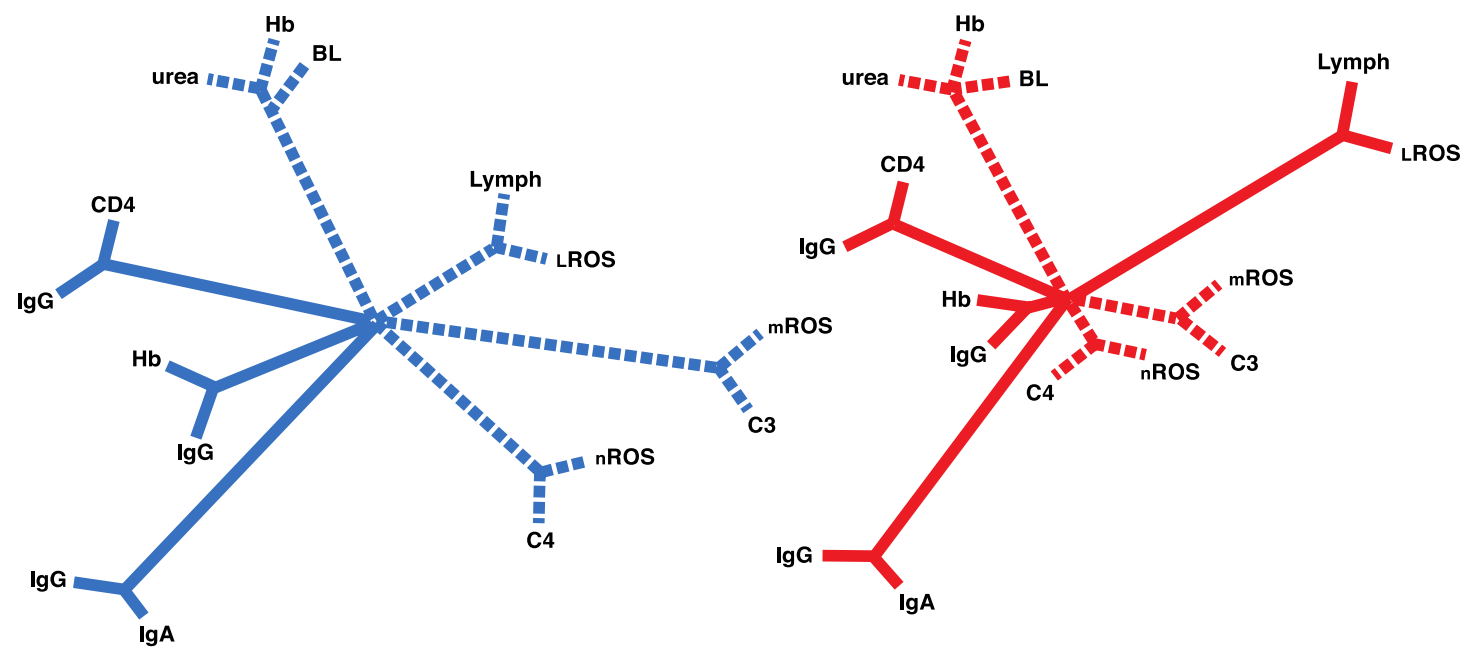

\section{B Minimally Invasive}

Changes in markers of haemodilution (urea and blood loss) after open surgery showed negative correlation with Haemoglobin, and this correlation was similar in VATS patients. Also, IgG and CD4 T lymphocyte changes showed close correlations in both surgical groups 2 days post surgery. In contrast, the correlation between haemodilution (Haemoglobin) and IgG changes observed 2 days after open surgery was much lower in VATS patients, indicating that the lower haemodilution in VATS patients had less impact on IgG levels after surgery. Correlations between IgG and IgA changes were similar after VATS and open surgery, indicating similar production/consumption in both patient groups 2 days after surgery.

On the right of each dendrograph is shown a negative correlation between lymphocyte number and lymphocyte ROS 2 days after open surgery, but a positive correlation in VATS patients. This supports findings that suggest that higher ROS levels are cytotoxic in lymphocytes. Differences between minimally invasive and open surgery in monocyte and neutrophil ROS were observed, compared with Complement (C3 and C4) 7 days after surgery. Less negative correlations between $\mathrm{C} 3$ and $\mathrm{C} 4$ and phagocyte ROS were detected in VATS patients indicating higher connectivity between opsonins and immune signalling after minimally invasive surgery. Pulmonary surgery was associated with increases in circulating complement $\mathrm{C} 3$ and $\mathrm{C} 4$, one week after surgery (Table 1). But this change was more gradual than immunoglobulin changes (Fig 2) and acute phase reactants [10], and C4 release was slightly reduced in VATS patients (Fig 2A). Increased C3 and C4 following surgery could indicate a slow onset "acute-phase" response. C3 and C4 may also represent a renal response to IL-6 released as a result of trauma, particularly muscle damage $[23,24]$ which is lower in minimally invasive procedures. 
Also, in a small preliminary study of circulating inflammatory and endothelial biomarker in part of the study group, increases in TNF receptor were observed (Table 2), which were smaller and slower than the rise in IL-6 levels (which peaked in these patients 4-12h after surgery). The kinetics of TNFR-II increases (Fig 3B) resembled C4 changes (Fig 3A) and these may be part of homeostatic responses to inflammatory stimuli elicited by surgery. Elevated circulating TNFR-II also correlates with progression of systemic inflammatory responses to sepsis and mortality [25]. A cellular target associated with metastatic progression is P-selectin [19], and the gradual TNFR-II increase we observed indicated greater endothelial cell adhesion following open surgery, with potential activation of $\mathrm{P}$-selectin associated metastatic signals. There is an increasing interest in incorporating these novel metabolomic biomarkers into clinical trials in cardiovascular medicine. In summary, in this study, we analysed changes in immune opsonins and inflammatory/endothelial biomarkers in individual patients following surgery. Decreases in immunoglobulins indicated a period of immune suppression, and complement and endothelial biomarkers suggested an acute phase/inflammatory response following pulmonary surgery. These changes were observed in both open surgery and minimally invasive surgery patients. In immunoglobulin turnover and blood loss, advantages of the minimally invasive route were detected in the immediate post operative period. Our preliminary findings of changes in the inflammatory marker TNFR-II and in the delayed acute response associated with C4 release followed earlier and greater increases in acute phase reactants CRP and IL-6. However, when minimally invasive and open surgery were compared, these complement and TNFR-II changes showed much less difference than acute phase changes in the same cohort. High correlations between humoral and cellular immune responses and haemodynamic markers were detected following surgery, and there was a shift towards more positive correlations in certain immune parameters following minimally invasive surgery, suggesting a closer immune connectivity in patients undergoing minimally invasive surgery.

The immediate postoperative period represents an opportunity for the use of therapeutic agents modulating immunological, acute phase and vascular signals which influence short term and long term survival. However, surgery is associated with substantial changes in these networks. This study supports the hypothesis that a decreased release of local trauma mediators, acute phase reactants, cellular immune and vascular responses all contribute to improved outcome and survival in minimally invasive surgery. Imaging techniques to detect local stress mediators will help define trauma signalling pathways at the site of surgery.

\section{ACKNOWLEDGEMENTS}

This study was undertaken as part of a postgraduate surgery study (SRC, supervised by WW). WW and SRC designed the clinical trial, performed the surgery and provided samples. HAL was involved in scientific design and assay performance and HAL and DR performed the data analysis and wrote up the study. Dr PL Yap provided immunoglobulin and complement determinations. We are grateful to Giuliana Rotondo for preparation of the dendrograph. Conflicts of interest: None declared. This work was supported by reagents grants from Chest Heart \& Stroke Scotland and the Melville Trust for the Care and Cure of Cancer. 


\section{REFERENCES}

[1] Michils, A.;Lambert, J.P.;Yernault, J.C.;Fabry, V.;Gossart, B., Duchateau, J. Fine tuning of epitopic dominance induced by lung cancer on the IgG response to bovine betalactoglobulin: towards a paraneoplastic immune marker. Cancer. 1996, 77, 657-664.

[2] Ceppa, D.P.;Kosinski, A.S.;Berry, M.F.;Tong, B.C.;Harpole, D.H.;Mitchell, J.D.;D'Amico, T.A., Onaitis, M.W. Thoracoscopic lobectomy has increasing benefit in patients with poor pulmonary function: a Society of Thoracic Surgeons Database analysis. Ann. Surg. 2012, 256, 487-493.

[3] Leaver, H.A.;Craig, S.R.;Yap, P.L., Walker, W.S. Lymphocyte responses following open and minimally invasive thoracic surgery. Eur. J. Clin. Invest. 2000, 30, 230-238.

[4] Ng, C.S.;Wan, S.;Hui, C.W.;Wan, I.Y.;Lee, T.W.;Underwood, M.J., Yim, A.P. Video-assisted thoracic surgery lobectomy for lung cancer is associated with less immunochemokine disturbances than thoracotomy. Eur. J. Cardio-thoracic surgery. 2007, 31, 83-87.

[5] Nosotti, M.;Rosso, L.;Mendogni, P.;Palleschi, A.;Tosi, D.;Bonara, P., Santambrogio, L. Leukocyte subsets dynamics following open pulmonary lobectomy for lung cancer: a prospective, observational study. Interact. Cardiovasc. Thorac. Surg. 2011, 13, 262-266.

[6] Pu, Q.;Ma, L.;Mei, J.D.;Zhu, Y.K.;Che, G.W.;Lin, Y.D.;Wu, Z.;Wang, Y.;Kou, Y.L.;Yang, J.J., Liu, L.X. [Immune functions of patients following lobectomy for lung cancers: a comparative study between video-assisted thoracoscopic surgery and posterolateral thoracotomy]. Sichuan Da Xие Xие Bao Yi Xue Ban. 2013, 44, 126-129.

[7] Friedrich, I.;Silber, R.;Baumann, B.;Fischer, C., Holzheimer, R. IgM-enriched immunoglobulin preparation for immunoprophylaxis in cardiac surgery. Eur. J. Med. Res. 2002, 7, 544.

[8] Pilz, G.n.;Appel, R.;Kreuzer, E., Werdan, K. Comparison of early IgM-enriched immunoglobulin vs polyvalent IgG administration in score-identified postcardiac surgical patients at high risk for sepsis. CHEST Journal. 1997, 111, 419-426.

[9] Ytting, H.;Christensen, I.J.;Basse, L.;Lykke, J.;Thiel, S.;Jensenius, J.C., Nielsen, H.J. Influence of major surgery on the mannan-binding lectin pathway of innate immunity. Clin. Exp. Immunol. 2006, 144, 239-246.

[10] Craig, S.R.;Leaver, H.A.;Yap, P.L.;Pugh, G.C., Walker, W.S. Acute phase responses following minimal access and conventional thoracic surgery. Eur. J. Cardiothoracic Surg. 2001, 20, 455463.

[11] Hu, J.K.;Zhou, Z.G.;Chen, Z.X.;Wang, L.L.;Yu, Y.Y.;Liu, J.;Zhang, B.;Li, L.;Shu, Y., Chen, J.P. Comparative evaluation of immune response after laparoscopical and open total mesorectal excisions with anal sphincter preservation in patients with rectal cancer. World J. Gastroenterol. 2003, 9, 2690-2694.

[12] Katayama, M.;Handa, M.;Araki, Y.;Ambo, H.;Kawai, Y.;Watanabe, K., Ikeda, Y. Soluble Pselectin is present in normal circulation and its plasma level is elevated in patients with thrombotic thrombocytopenic purpura and haemolytic uraemic syndrome. Br. J. Haematol. 1993, 84, 702-710.

[13] Roselli, M.;Mineo, T.;Martini, F.;Mariotti, S.;Ambrogi, V.;Spila, A.;D'Alessandro, R.;Basili, S.;Guadagni, F., Ferroni, P. Soluble selectin levels in patients with lung cancer. International J. Bio.l Markers. 2001, 17, 56-62.

[14] Sakamaki, F.;Ishizaka, A.;Handa, M.;Fujishima, S.;Urano, T.;Sayama, K.;Nakamura, H.;Kanazawa, M.;Kawashiro, T.;Katayama, M. Soluble form of P-selectin in plasma is elevated in acute lung injury. Am. J. Respir. Crit. Care Med. 1995, 151, 1821-1826.

[15] Glovsky, M.M.;Ward, P.A., Johnson, K.J. Complement determinations in human disease. Ann. Allergy Asthma Immunol. 2004, 93, 513-522; quiz 523-515, 605.

[16] Ramadori, G.;Van Damme, J.;Rieder, H., Meyer zum Buschenfelde, K.H. Interleukin 6, the third mediator of acute-phase reaction, modulates hepatic protein synthesis in human and mouse. Comparison with interleukin 1 beta and tumor necrosis factor-alpha. Eur. J. Immunol. 1988, 18, 1259-1264.

[17] Leen, C.L.;Yap, P.L., McClelland, D.B. Increase of serum immunoglobulin level into the normal range in primary hypogammaglobulinaemia by dosage individualization of intravenous immunoglobulin. Vox Sang. 1986, 51, 278-286.

[18] Whicher, J.T.;Perry, D.E., Hobbs, J.R. An evaluation of the Hyland laser nephelometer PDQ system for the measurement of immunoglobulins. Ann Clin Biochem. 1978, 15, 77-85. 
[19] Garcia, J.;Callewaert, N., Borsig, L. P-selectin mediates metastatic progression through binding to sulfatides on tumor cells. Glycobiology. 2007, 17, 185-196.

[20] Walker, W.S., Leaver, H.A. Immunologic and stress responses following video-assisted thoracic surgery and open pulmonary lobectomy in early stage lung cancer. Thorac. Surg. Clin. 2007, 17, 241-249, ix.

[21] Champault, G. [Has laparoscopic surgery reached its limits?]. J Chir (Paris). 1996, 133, 356-357.

[22] Cook-Mills, J.M. VCAM-1 signals during lymphocyte migration: role of reactive oxygen species. Mol. Immunol. 2002, 39, 499-508.

[23] Semple, S.J.;Smith, L.L.;McKune, A.J.;Hoyos, J.;Mokgethwa, B.;San Juan, A.F.;Lucia, A., Wadee, A.A. Serum concentrations of $\mathrm{C}$ reactive protein, alpha1 antitrypsin, and complement (C3, C4, C1 esterase inhibitor) before and during the Vuelta a Espana. Br. J. Sports Med. 2006, 40, 124-127.

[24] van de Vyver, M., Myburgh, K.H. Cytokine and satellite cell responses to muscle damage: interpretation and possible confounding factors in human studies. J. Muscle Res. Cell Motil. 2012, 33, 177-185.

[25] Punyadeera, C.;Schneider, E.M.;Schaffer, D.;Hsu, H.Y.;Joos, T.O.;Kriebel, F.;Weiss, M., Verhaegh, W.F. A biomarker panel to discriminate between systemic inflammatory response syndrome and sepsis and sepsis severity. J. Emerg. Trauma Shock. 2010, 3, 26-35. 